\title{
The Atmospheres of the Giant Planets
}

By Dr. Arthur Ader and Dr. V. M. Slupher, Lowell Observatory and the University of Michigan

7 HE present paper is concerned with the results 1 of an investigation carried out to ascertain the extent to which the methane molecule $\left(\mathrm{CH}_{4}\right)$ is responsible for the spectrum of the major planets.

In order to seeure intensities of absorption comparable with those obtained in the planets, a pathlength of two thousand metre-atmospheres was employed. The spectrum of a source of continuous radiation shining through the gas was secured with a glass Hilger $E$-I spectrograph, and the photographs cover the region from the violet to the infra-red.

A survey of the band spectrum of gaseous methane is above all characterised by the prominent overtone sequence of the $\nu_{3}$ fundamental at $3 \cdot 3 \mu$. This sequence coincides precisely with the outstanding group of absorption bands in the spectra of the giant planets. system of combination bands. Not all of the methane bands showing in the spectrum of the outer planets have as yet been detected in the laboratory, however, inasmuch as the absorption columns in Neptune and Uranus are an order of magnitude greater than the laboratory path-length described above. In conformity with expectation, the planetary bands of the type $n v_{3}+v_{i}$ which have been duplicated in the laboratory involve the smaller values of $n$. The present absence of complete duplication is, of course, no obstacle to the identification of the planetary bands. The fact that there exist but four normal modes of vibration of the methane molecule, and that the selection rules governing the band spectrum prohibit the appearance of the frequencies $p v_{1}+q \nu_{2}$, means that there is a minimum of overlapping in

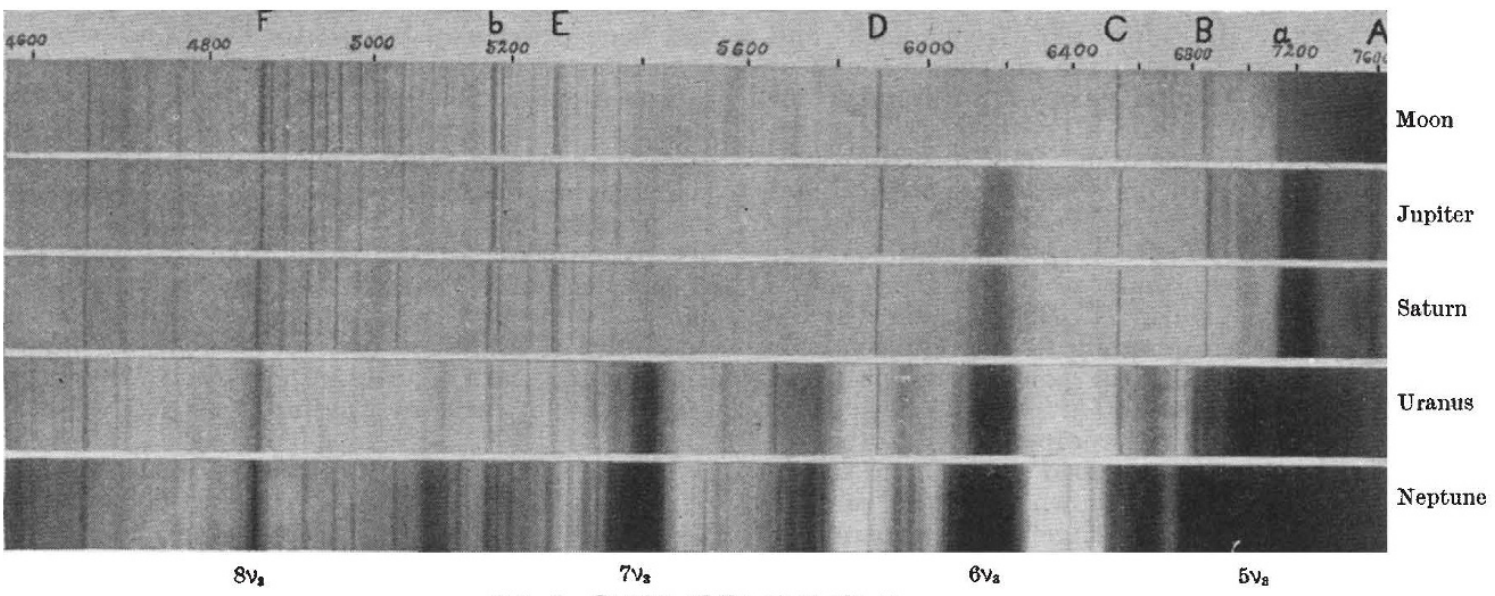

Fit. 1. Spectra of the giant planets.

The latter is shown in Fig. 1, in which the series of bands $5 v_{3}, 6 v_{3}, 7 v_{3}$ and $8 v_{3}$ are immediately discermible. It is especially gratifying to observe that the band which is superimposed upon $\mathrm{H}_{\beta}$ is included amongst the members of this group.

The harmonic nature of the above sequence is nicely displayed by the curve of Fig. 2. Extrapolation shows that planetary absorption may be expected at about $4410 \mathrm{~A}$. (extremely weak) and at about $11,540 \mathrm{~A}$. (extremely intense). The $4410 \mathrm{~A}$. band is with very little doubt to be identified as the Neptune band reported by Slipher ${ }^{1}$ at $44 \operatorname{lm} \mu$. This is the ninth harmonic of $\nu_{3}$, and Neptune alone amongst the major planets has sufficient methane in the absorbing layers of its atmosphere to register it.

In virtue of the fact that this sequence of rotationvibration bands is the most prominent in the entire band spectrum of the molecule, it is to be expected that its members will combine with the other fundamental vibrations $\left(v_{1}=2,915 \mathrm{~cm} .^{-1}, v_{2}=1,520 \mathrm{~cm} .^{-1}\right.$, $v_{4}=1,304 \mathrm{~cm}^{-1}$ ) in the production of the system of absorption bands $n v_{3}+v_{i}$, where $i$ may equal 1, 2 or 4 ; and that these bands will be amongst the next strongest in the spectrum. This is indeed the case, for the bands which appear in the laboratory and planetary spectra of methane (other than the group $n \nu_{3}$ ) are for the most part members of this the consequential spectral regions, and therefore bui very small uncertainty in the correlation. The astronomical data employed in the analysis are those of the extensive investigations of V. M. Slipher of the Lowell Observatory. Reference should be made to the Lowell Observatory Bulletins, and the Monthly Notices of the Royal Astronomical Society for descriptions and illustrations of the absorption bands in the remarkable spectra of the giant planets ${ }^{2}$. The spectrum has been recorded into the infra-red as far as $10,000 \mathrm{A.}^{3}$.

In the identification given below, the bands which have thus far been duplicated in the laboratory are followed by asterisks.

Identification of the Planetary Methane Bands of the Type $n v_{3}$

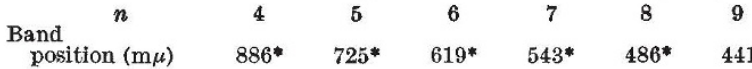

Identiflcation of the Planetary Methane Bands of the Type $n v_{s}+\nu_{i}$ $\begin{array}{lccccc}i=1 & n & 4 & 5 & 6 \\ & \text { Band position }(\mathrm{m} \mu) & 861^{*} & 702^{*} & 595 & 521\end{array}$

$\begin{array}{lcccc}i=2 & n & 4 & 5 & 6 \\ & \text { Band position }(\mathrm{m} \mu) & 782 * & 656 & 568 \\ i=4 & & 5 & 6 & 7\end{array}$

$\begin{array}{lccccc}i=4 & 5 & 6 & 7 & 8 \\ & \text { Band position }(\mathrm{m} \mu) & 668^{*} & 576 & 509 & 459-460\end{array}$

$\nu_{4}$ is a very low frequency oscillation, and therefore 
only its high harmonies will invade the photographic region of the spectrum. Consequently, we should expect the methane bands $m v_{4}$ in the planets to be weak ones. This is indeed the case. The harmonic nature of the sequence is strikingly brought out in Fig. 3.

Identiflcation of the Planetary Methane Bands of the Type $n v_{4}$

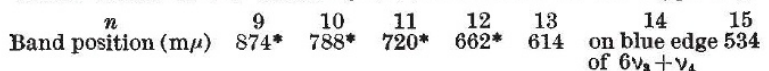

An idea of the complexity of the fine structures of the methane absorption bands may be gained

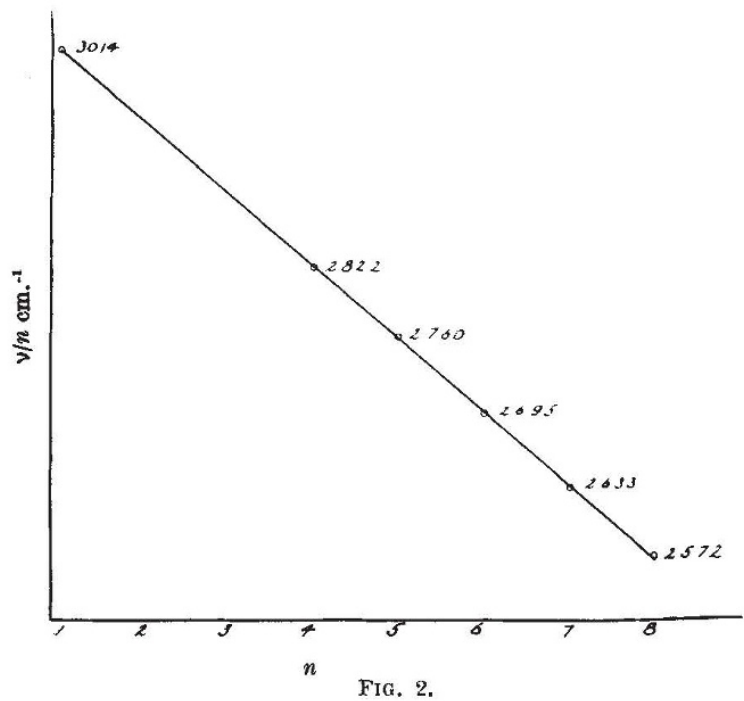

from the following examination of the harmonics of $v_{3}$.

The $v_{3}$ mode of vibration of the methane molecule possesses three degrees of freedom. An analysis by Dennison and Ingram ${ }^{4}$ based upon this fact in conjunction with the tetrahedral symmetry of the molecular force field has shown that the vibrational energy levels $n \nu_{3}$ are multiple with a multiplet separation which is very small in comparison with the fundamental frequency; in fact, of the same order of magnitude as the rotational structure. Thus, when $n$ is the order of the harmonic, approximately $\frac{1}{4}(n+1)(n+2)$ bands superpose to form a single composite absorption band $n \nu_{3}$. Consequently, such a band as the $7 v_{3}$ one in the planets is actually a superposition of some eighteen bands. It is not sur- prising, therefore, when such a band proves difficult of resolution.

A predominantly hydrncarbon nature seems not at all unlikely for the giant planets. Aside from the above very probable identification of methane, this conclusion is also indicated by the fact that the mean densities of the giants are in a class with the densities of most organic liquids. Furthermore, it is not surprising that methane should stand out so prominently, inasmuch as its vapour pressure at the extremely low temperatures which prevail in the atmospheres of the outer planets is far in excess of the vapour pressures of any of the other hydrocarbons apt to exist there.

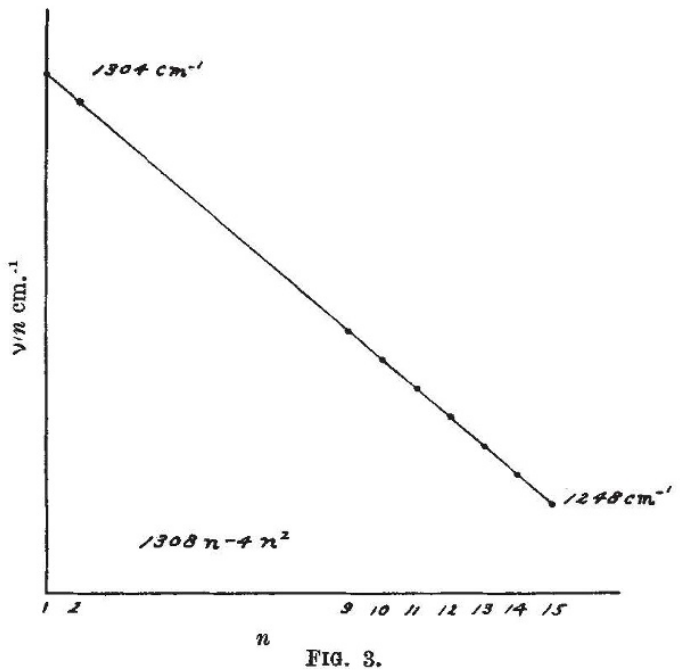

It is a curious and noteworthy fact that the two constituents, of the existence of which in the planetary atmospheres we are now fairly certain, namely, methane and ammonia, are saturated with hydrogen. This fact, taken in conjunction with the very stable natures of these two compounds, may signify that collision reactions in the atmospheres of the giant planets have continued over vast periods of time in a progression of the atmospheric constitu. ents towards maximum stability relative to the physical conditions which prevail in the planetary atmospheres.

${ }^{1}$ Lowell Obs. Bull., No. 42.

${ }^{2}$ Mon. Not. Roy. Ast. Soc, 93, No, 9; 1933

${ }^{3}$ Mon. Not. Roy. Ast. Soc., 93, No. 9, Plate 15, Fig. 3; 1933.

Phys. Rev., 36, 1451; 1930.

\section{Scientific Studies of Noise*}

$\mathrm{T}$ $\mathrm{HE}$ subject of noise control is being actively studied scientifically at the U.S. Bureau of Standards, the Heinrich Hertz Institute in Berlin, and at the National Physical Laboratory in Great Britain, while a number of firms have developed noise-measuring apparatus, means for reducing noiselevels, and materials and methods for isolating vibration. Literature tells us that the noise problem is by no means new, but in addition to the old sources of noise, civilisation brings in new sources; there is evidence of a growing noise-consciousness in the

* Summary of an address delivered by Dr. G. W. C. Kaye, superintendent of the Physics Department, National Physical Laboratory, before the Science and Noise Section of the Conference of the AntiNoise League held at Oxford on July 14 public, possibly due to increased nerve strain and reduced tolerance, and increased sense of the social value of noise reduction.

The problem is being considered by a Committee of the British Standards Institution, with the view of arriving at an acceptable definition of noise and methods of measurement.

From the general point of view, any type of noise may annoy someone, the nuisance value depending on the marginal or 'last straw' effect, the latter being largely personal. Although considerable noise can be tolerated, particularly when self-generated, Bartlett is of the opinion that the cumulative effect of some slight adjustments by a worker may in time produce 